\title{
Experimental evolution in Chlamydomonas. I. Short-term selection in uniform and diverse environments
}

\author{
GRAHAM A. C. BELL* \\ Redpath Museum, McGill University, 859 Sherbrooke Street West, Montreal, Quebec, Canada H3A $2 K 6$
}

\begin{abstract}
Genetically diverse cultures of the unicellular chlorophyte Chlamydomonas were selected for about 50 generations in a heterogeneous environment, comprising eight different culture media, and in a uniform environment consisting of the average of the eight different media. The genetic variance of fitness, either across or within media, was reduced much more severely in the uniform than in the heterogeneous environment. This effect appears to have been caused by the obstruction of directional selection in the heterogeneous environment, leading to the retention of a greater quantity of genetic variance in the short term.
\end{abstract}

Keywords: Chlamydomonas, environmental heterogeneity, experimental evolution, genetic variation, selection.

\section{Introduction}

Selection in a uniform environment is expected to increase adaptation by causing the loss of all types except one. There are certain special cases in which this conclusion does not hold, for example heterozygote advantage in sexual diploid organisms. However, the most general cause of the maintenance of substantial quantities of genetic variance may be that environments are not uniform. Diversity may be maintained in heterogeneous environments, if selection favours different types at different sites. Tilman (1982) has defined the conditions in which environmental heterogeneity will permit species coexistence and validated these conditions experimentally. Rosenzweig (1995) has summarized the case for believing that species diversity can be understood largely in terms of habitat diversity. At the level of genetic variation within populations, the evidence (reviewed by Hedrick, 1986) is not as clear. It can be shown theoretically that selection in a coarse-grained environment, in which individuals live all their lives in a single habitat and their offspring disperse among habitats at the beginning of each generation, can lead to a stable equilibrium of allele frequencies (Levene, 1953). This conclusion requires soft selection, created through the indepen-

*E-mail: graham_bell@maclan.mcgill.ca dent regulation of density at each site; hard selection, created through the regulation of density at the level of the whole population, does not maintain genetic diversity (Dempster, 1955). Models of selection in heterogeneous environments have been reviewed and generalized by Maynard Smith \& Hoekstra (1980) and by Via \& Lande (1985). The elaboration of the theory has not been matched by a similar experimental effort. The only experiment that is directly relevant to the Levene model and its descendants is that reported by Haley \& Birley (1983), in which allelic diversity at allozyme loci was measured in laboratory populations of Drosophila with up to three kinds of substrate available for oviposition. Other experiments involve environmental variation, but not the coarse-grained spatial heterogeneity envisaged by the Levene model; for example, fine-grained spatial heterogeneity (e.g. Minawa \& Birley, 1978) or temporal variation (e.g. Yamazaki et al., 1980). What experimental evidence is available has been reviewed by Hedrick (1986). All of these experiments concern the maintenance of genotypic diversity, as indicated by the frequencies of allozyme markers; the crucial predictions of the model, however, concern the persistence of genetic variance in fitness. Environmental heterogeneity should obstruct directional selection and retard the loss of genetic variance. Moreover, genetic variance may be permanently maintained under soft selec- 
tion. The experiment reported in this paper is the first attempt to test these predictions experimentally.

\section{Materials and methods}

\section{Base population}

The experimental organism is the unicellular chlorophyte, Chlamydomonas reinhardtii. Basic biology and laboratory procedures not referenced here are given by Harris (1989). Chlamydomonas reinhardtii is haploid and heterothallic. It can be propagated vegetatively in minimal medium for as long as required, or induced by nitrogen starvation to form gametes and mate. Genetic and environmental variation in fitness are described by Bell $(1990,1991)$. A single mating-type plus isolate (CC-1010) was crossed with four mating-type minus strains (CC-1009, CC-410, CC-1418 and CC-1952), isolating $6 \mathrm{mt}^{+}$and $6 \mathrm{mt}^{-}$spores (vegetative cells) from each cross, to form a base population comprising a mixture of 48 spores. CC-1010 and CC-1009 are fullsibs; the other three $\mathrm{mt}^{-}$strains are independent isolates from widely separated localities.

\section{Environments}

Eight environments were constructed as factorial combinations of three macronutrients using Bold's liquid minimal medium as a basis: nitrate and phosphate (each 100 per cent or 25 per cent of standard concentration) and bicarbonate (zero or $250 \mathrm{mg} \mathrm{L}^{-1}$ sodium bicarbonate). This environmental variation is known to cause substantial differences both in average growth rate and in relative growth rates (see Bell, 1992). Experimental design is shown schematically in Fig. 1. The unit of growth was $40 \mathrm{~mL}$ of culture medium in a screw-top glass tube. The diverse environment consisted of five replicate tubes of each of the eight media, arranged randomly in a rack. Each rack thus represented a coarse-grained environment, within which each set of five tubes represented a particular habitat, defined as a combination of macronutrient concentrations. The environment was coarse-grained because the cultures are confined to the tubes throughout each growth cycle and are unable to move from tube to tube. The uniform environment consisted of 40 replicate tubes of a single medium, made up by mixing equal quantities of each of the eight different media. Each rack thus represented an environment that lacked spatial heterogeneity, consisting exclusively of a single habitat. Cultures were allowed to grow for 14 days under continuous fluorescent light.

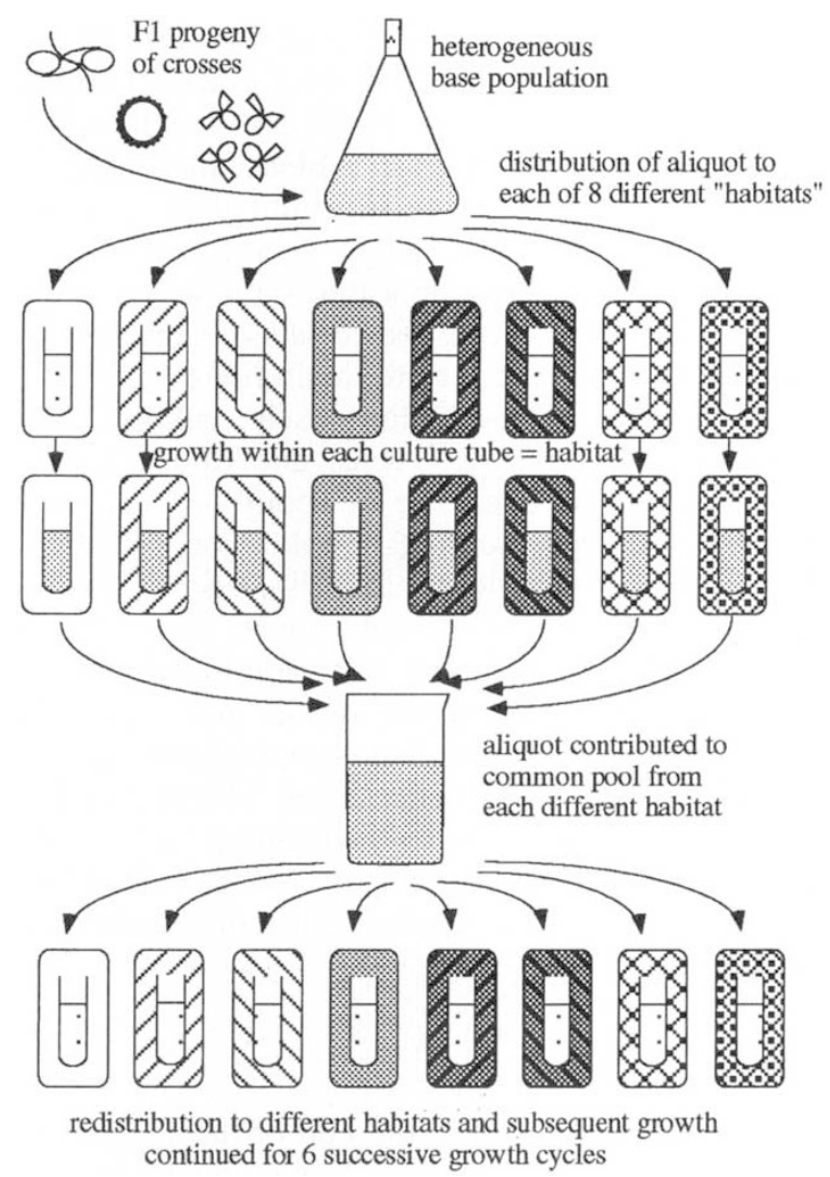

Fig. 1 Schematic representation of the experiment. The diagram shows the population structure of the 'diverse' environment. The 'uniform' environment was organized in a similar fashion, except that all culture tubes held the same growth medium.

\section{Serial transfer procedure}

After growth, an aliquot was taken from all cultures within a treatment, and these aliquots were mixed together. Hard and soft selection were imposed through the way in which the aliquots were taken. Hard selection involved taking a fixed volume $(1 \mathrm{~mL})$ from each tube. Soft selection involved taking a fixed number of cells from each tube, which was accomplished in practice by taking a fixed volume of $1 \mathrm{~mL}$ from each tube after the culture had been diluted to a fixed optical density. The mixture formed in this way was then diluted to a fixed optical density, both in the hard and in the soft selection lines, and each fresh culture tube was inoculated with $1 \mathrm{~mL}$ of this diluted mixture. After 14 days the procedure was repeated.

The experiment thus comprised four treatments, formed by combinations of two levels of environ- 
mental heterogeneity (uniform and diverse) and two modes of selection (hard and soft). Each treatment was physically represented by 40 culture tubes held in racks grouped together as a block, the entire set of 160 culture tubes being surrounded by a border row and laid out on a single growth shelf. This arrangement was then duplicated on a second shelf, so that there were two replicate sets of experimental lines, which were transferred on alternate weeks.

The inoculum used for each culture tube contained about $0.6 \times 10^{6}$ cells, and after 14 days' growth these had given rise to about $7-8 \times 10^{7}$ cells in each tube, representing about seven doublings. (Growth was much slower than is possible because the tubes, packed tightly into racks, were heavily shaded.) The experiment was continued for six cycles, and consequently the results are relevant to the sorting of variation in large populations over the short term of about 50 generations. The populations are in vegetative growth throughout; sex is not required by the theory being tested (see Strobeck, 1974) and was not induced at any point during the experiment.

\section{Assay procedure}

The lines were assayed at the end of the experiment by isolating spores and measuring growth in pure culture, measured as optical transmittance at $665 \mathrm{~nm}$ on a Bausch and Lomb Spec-20 digital spectrophotometer. In each replicate set of experimental lines, eight spores were sampled at random from each treatment and also from the base population, which had been stored during the experiment on solid medium. Each of the 40 spores (i.e. eight from each of the four selection treatments and the base population) was grown in all eight culture environments, under conditions which approximated as closely as possible those during selection. The character scored was the growth of $1 \mathrm{~mL}$ of standard inoculum after 14 days; that is, the character scored was identical with the character selected and represents fitness in the circumstances of the experiment. The assay was blocked in time, each genotype-environment combination being tested once, and then all being subsequently tested again. This is a conservative procedure, because the error variance includes both the fundamental between-replicate-culture variance and also any variance attributable to variation in culture conditions through time. The entire assay was then repeated, using a second sample of eight random spores from each selection line and from the base population; the first and second assays are referred to as 'samples'. The entire assay thus comprised five treatments (four selection treatments plus the base population) $\times$ two lines (replicate experiments) $\times$ two samples (of eight spores from each line) $\times$ eight spores per sample $\times$ eight environments (culture media) $\times$ two replicates of each genotype-environment combination $=2560$ cultures.

\section{Analysis}

To characterize genetic variation across environments, the data were analysed by two-way analysis of variance using the GLM procedure in SAS, to yield estimates of mean squares attributable to genotype (7 d.f.), environment ( 7 d.f.), genotype-environment interaction (49 d.f.) and replication (error) (64 d.f.). Variance components were calculated by equating observed with expected mean squares; these estimates were sometimes negative because of sampling error. Genetic variation within environments was analysed by single-classification analysis of variance using the GLM procedure in SAS, to yield estimates of genetic variance with 7 d.f. In both cases, $F$-tests were used to evaluate the probability $P$ that the estimates differed from zero by chance alone, treating genotypes as random effects and environments as fixed effects.

\section{Results}

\section{Genetic variance over environments}

Mean performance (growth at 14 days), phenotypic variance and the components of phenotypic variance are shown in Table 1 . Mean performance increased through selection, as expected, in the uniform treatment. With hard selection, the response of fitness in units of phenotypic standard deviations of the base population was $+0.76(t=2.16$, d.f. $=30, P<0.05)$ and $+0.72(t=2.27, P<0.05)$ in the two replicate lines. With soft selection, the responses were +0.70 $(t=1.86, \quad 0.1>P>0.05) \quad$ and $+0.56 \quad(t=1.74$, $0.1>P>0.05)$. Selection was ineffective in increasing mean fitness in the diverse treatment, either under hard selection (responses +0.01 and +0.40 , both with $P>0.5$ ) or under soft selection (responses +0.30 and +0.37 , both with $P>0.2$ ).

There was abundant genetic variance in the base population, as required for the theory to be tested. Environmental variance is displayed by the base population and by all selection lines, showing that the manipulation of culture conditions was successful in creating a heterogeneous environment. Genetic variance of fitness disappears in the uniform treatments, with the exception of one set of spores 
Table 1 Genetic variance over environments

\begin{tabular}{|c|c|c|c|c|c|c|c|c|c|}
\hline \multirow[b]{2}{*}{ Environment } & \multirow[b]{2}{*}{ Selection } & \multirow[b]{2}{*}{$\mathrm{L}$} & \multirow[b]{2}{*}{$\mathrm{S}$} & \multicolumn{6}{|c|}{ Variance components } \\
\hline & & & & Mean & $P$ & $G$ & $E$ & $G E$ & $e$ \\
\hline \multirow[t]{4}{*}{ Initial } & \multirow[t]{4}{*}{ None } & 1 & 1 & 505.7 & 6419 & $1577^{* * *}$ & $751^{* *}$ & $1418^{* *}$ & 2958 \\
\hline & & 1 & 2 & 472.4 & 9261 & $635^{* *}$ & $652^{*}$ & $4847 * *$ & 3317 \\
\hline & & 2 & 1 & 494.8 & 9659 & $2090^{* * *}$ & $824 *$ & $3392^{* * *}$ & 3725 \\
\hline & & 2 & 2 & 451.6 & 8719 & $1802^{* * *}$ & $2548^{* * *}$ & $1692 * *$ & 3204 \\
\hline \multirow{4}{*}{ Uniform } & \multirow{4}{*}{ Hard } & 1 & 1 & 566.1 & 5443 & -165 & $2088^{* * *}$ & 383 & 3367 \\
\hline & & 1 & 2 & 545.6 & 9552 & 131 & $6437^{* * *}$ & -4 & 3764 \\
\hline & & 2 & 1 & 552.4 & 4962 & -9 & $1523^{* * *}$ & 311 & 3048 \\
\hline & & 2 & 2 & 531.1 & 5846 & 99 & $1745^{* * *}$ & 135 & 4086 \\
\hline \multirow[t]{4}{*}{ Uniform } & \multirow[t]{4}{*}{ Soft } & 1 & 1 & 547.3 & 9251 & 114 & $6066^{* * *}$ & -990 & 4061 \\
\hline & & 1 & 2 & 553.9 & 9866 & 232 & $8115^{* * *}$ & -864 & 3362 \\
\hline & & 2 & 1 & 515.9 & 7294 & $1748^{* * *}$ & $925 * * *$ & $1523 * *$ & 3425 \\
\hline & & 2 & 2 & 538.6 & 5421 & -31 & $2255^{* * *}$ & -956 & 4409 \\
\hline \multirow{4}{*}{ Diverse } & \multirow[t]{4}{*}{ Hard } & 1 & 1 & 505.2 & 9379 & $3675^{* * *}$ & $3382^{* * *}$ & $700^{*}$ & 2461 \\
\hline & & 1 & 2 & 473.3 & 9978 & $2985^{* * *}$ & $2694^{* * *}$ & $1685^{* *}$ & 3298 \\
\hline & & 2 & 1 & 493.8 & 5004 & $274^{* * *}$ & $1165^{* * *}$ & $1162^{* *}$ & 2583 \\
\hline & & 2 & 2 & 477.0 & 6523 & $458^{* *}$ & $2110^{* * *}$ & $792^{*}$ & 3473 \\
\hline \multirow[t]{4}{*}{ Diverse } & \multirow[t]{4}{*}{ Soft } & 1 & 1 & 512.0 & 9784 & $2191^{* * *}$ & $3534 * * *$ & 1190 & 3554 \\
\hline & & 1 & 2 & 519.5 & 13045 & $3501^{* * *}$ & $6350^{* * *}$ & 630 & 3732 \\
\hline & & 2 & 1 & 512.4 & 7268 & $436^{* *}$ & $1804^{* * *}$ & 822 & 4477 \\
\hline & & 2 & 2 & 504.3 & 8549 & $267^{* * *}$ & $1945^{* *}$ & $3513 * * *$ & 3113 \\
\hline
\end{tabular}

Data are given for the two samples (S) scored from each of the two replicate selection lines (L) for each treatment.

Variance components are attributable to: phenotype, $P$; genotype, $G$; environment, $E$; genotype-environment interaction, $G E$; and replication, $e$. The probability that an estimate differs from zero by chance alone is summarized in the table as:

${ }^{* * *} P<0.001 ;{ }^{* *} 0.001<P<0.01 ;{ }^{*} 0.01<P<0.1$; no asterisk, $P>0.1$.

from a soft selection line. Substantial and highly significant amounts of genetic variance are retained in all the diverse treatments.

\section{Genetic variance within environments}

The genetic variance of fitness in each environment (culture medium) considered separately is indicated in Table 2. There is again much variation in the base population: $23 / 32$ estimates are significant at $P<0.1$. This variation is greatly attenuated by selection in the uniform treatments: only $7 / 64$ estimates of genetic variance are significant at $P<0.1$, a proportion that scarcely differs from that expected by chance. This attenuation is much less marked in the diverse treatments, where 30/64 estimates are significant at $P<0.1$. Even on this crude basis, the difference between uniform and diverse environments is highly significant $\left(\chi_{1}^{2}=20.1, \quad P<0.001\right)$. The frequency distribution of genetic variance within environments is shown for each treatment in Fig. 2.
The mean value of estimates of genetic variance in the base population was $4384(\mathrm{SD}=3129, \mathrm{SE}=553$, $n=32$ ). Selection in the uniform environment had virtually eradicated genetic variance by the end of the experiment, whether selection was hard $($ mean $=225, \quad \mathrm{SD}=1414, \quad \mathrm{SE}=250) \quad$ or $\quad$ soft (mean $=194, \quad S D=3079, \quad S E=544$ ). Substantial quantities of genetic variance were retained in the diverse treatment, again regardless of whether selection was hard $($ mean $=2933, \mathrm{SD}=3142, \mathrm{SE}=555)$ or soft $($ mean $=3106, \mathrm{SD}=3856, \mathrm{SE}=682)$. The quantity of genetic variance remaining after selection in the diverse treatment is probably somewhat less than that originally present in the base population (for hard selection, $t=1.85$, d.f. $=62, P=0.077$; for soft selection $t=1.46, P=0.156$; combining results for hard and soft selection gives $\chi_{2}^{2}=8.84$, $P=0.02$ ). However, there is much less variance remaining after selection in the uniform treatment than after selection in the diverse treatment (for hard selection, $t=4.45, P<0.001$; for soft selection, $t=3.34, P=0.002$ ). 
Table 2 Genetic variance within environments

\begin{tabular}{|c|c|c|c|c|c|c|c|c|c|c|c|}
\hline \multirow[b]{2}{*}{ Environment } & \multirow[b]{2}{*}{ Selection } & \multirow[b]{2}{*}{ B } & \multirow[b]{2}{*}{$\mathbf{S}$} & \multicolumn{8}{|c|}{ Conditions } \\
\hline & & & & 1 & 2 & 3 & 4 & 5 & 6 & 7 & 8 \\
\hline Initial & None & $\begin{array}{l}1 \\
1 \\
2 \\
2\end{array}$ & $\begin{array}{l}1 \\
2 \\
1 \\
2\end{array}$ & $\begin{array}{l}* \\
* \\
*\end{array}$ & $\begin{array}{c}* * \\
* * * \\
* * \\
* *\end{array}$ & & $\begin{array}{c}* * \\
* * \\
* * \\
*\end{array}$ & $\begin{array}{l}* \\
* \\
*\end{array}$ & $\begin{array}{l}* * \\
* * \\
* \\
*\end{array}$ & * & $\begin{array}{c}* \\
* * \\
* * \\
*\end{array}$ \\
\hline Uniform & Hard & $\begin{array}{l}1 \\
1 \\
2 \\
2\end{array}$ & $\begin{array}{l}1 \\
2 \\
1 \\
2\end{array}$ & $*$ & $*$ & & & & & * & \\
\hline Uniform & Soft & $\begin{array}{l}1 \\
1 \\
2 \\
2\end{array}$ & $\begin{array}{l}1 \\
2 \\
1 \\
2\end{array}$ & $* *$ & & $* * *$ & & $*$ & & & $*$ \\
\hline Diverse & Hard & $\begin{array}{l}1 \\
1 \\
2 \\
2\end{array}$ & $\begin{array}{l}1 \\
2 \\
1 \\
2\end{array}$ & $*$ & $\begin{array}{l}* \\
* \\
*\end{array}$ & $\begin{array}{l}* * \\
* * *\end{array}$ & $\begin{array}{c}* * * \\
*\end{array}$ & & $\begin{array}{c}* \\
* \\
* *\end{array}$ & $\begin{array}{l}* \\
*\end{array}$ & $\begin{array}{l}* \\
* \\
*\end{array}$ \\
\hline Diverse & Soft & $\begin{array}{l}1 \\
1 \\
2 \\
2\end{array}$ & $\begin{array}{l}1 \\
2 \\
1 \\
2\end{array}$ & & $* *$ & $\begin{array}{c}* * \\
* \\
* *\end{array}$ & $\begin{array}{l}* \\
*\end{array}$ & & $\begin{array}{l}* \\
* \\
* \\
*\end{array}$ & $*$ & $\begin{array}{l}* * \\
* \\
*\end{array}$ \\
\hline
\end{tabular}

Significance levels indicated as in Table 1.

\section{Discussion}

A biologist sampling a population may collect individuals from a single habitat, or from the whole range of habitats occupied by the population. It will rarely be possible to say with confidence which of these two extremes is closer to reality. The results of this experiment show that the genetic variance of fitness tends to be conserved in heterogeneous environments, regardless of the scale of sampling. They therefore provide an experimental validation of the proposition that the genetic variance of fitness observed in natural populations can be attributed to variable selection in heterogeneous environments.

Genetic variance may be lost from the uniform treatments either because the populations become genetically uniform or because, although genetically diverse, their members express similar phenotypes. This was investigated by culturing spores from the selected populations in a series of exotic environments to which they had not previously been adapted (Table 3). The base population, as expected, expressed substantial genetic variance in these environments. This was generally lost from the uniform selection lines, suggesting that the lack of genetic variance reported from the eight selection environments reflected a real genotypic uniformity. However, in one of the two hard selection lines substantial genetic variance was expressed. This occurred in both samples and all three environments. In this case, it is possible that the phenotypic uniformity observed in the selection environments concealed substantial genotypic diversity.

In the Levene model of soft selection, variation may be stably conserved at equilibrium; under hard selection, the elimination of variation is slower than in a uniform environment, but no intermediate equilibrium value is attained. This experiment showed no detectable difference between hard and soft selection in heterogeneous environments. Moreover, the genotype-environment variance is not consistently greater under soft selection, and further analysis (not shown) shows that there is no tendency for genetic correlations (of fitness in two environments) to become negative; they become smaller positive in one block and larger positive in the other. There is thus no evidence that genetic variance is tending towards an equilibrium under soft selection. In similar studies involving growth in media with 
Fig. 2 Effect of uniform and diverse environments on quantity of genetic variance in fitness. For each selection treatment, the histogram shows the frequency distribution of the 32 estimates of within-environment genetic variance available ( 2 lines $\times 2$

samples $\times 8$ media). The histogram for the base population is repeated to facilitate comparison.
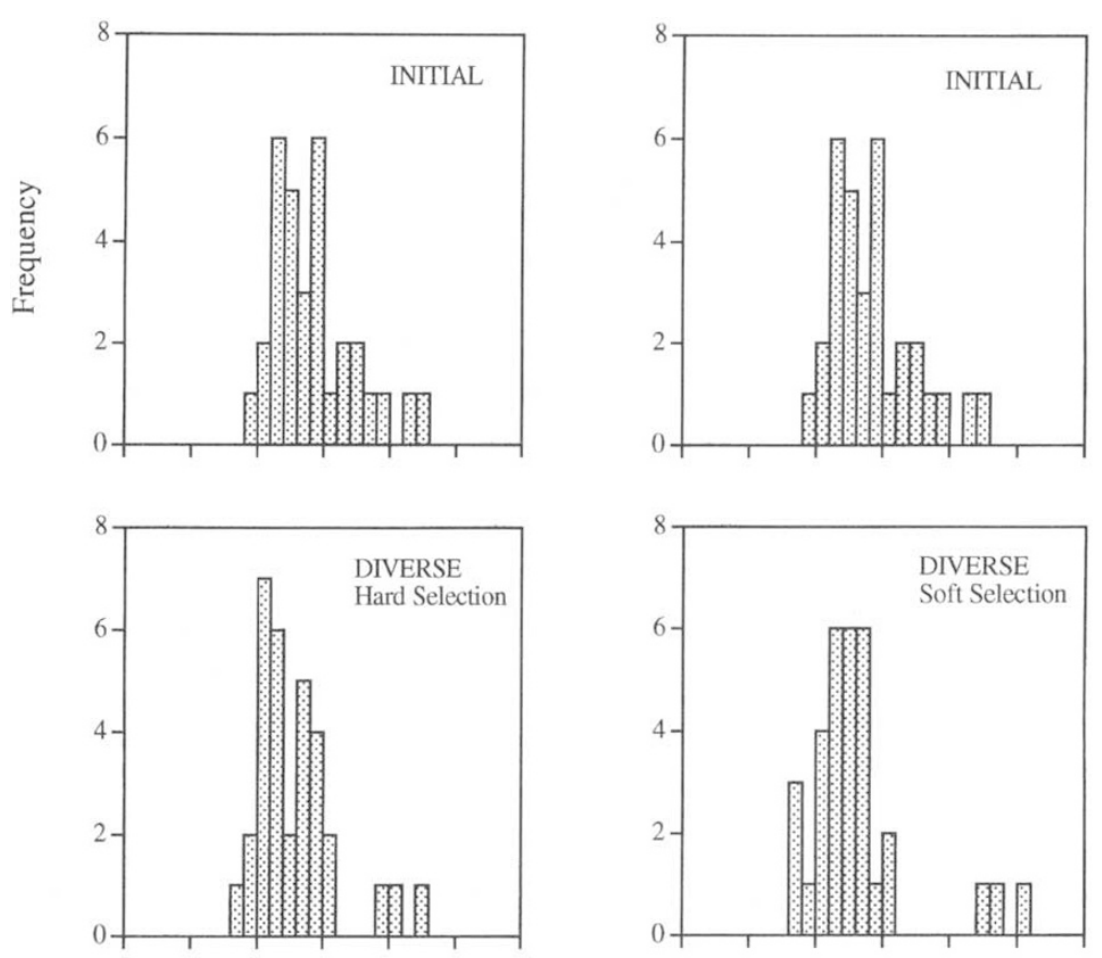

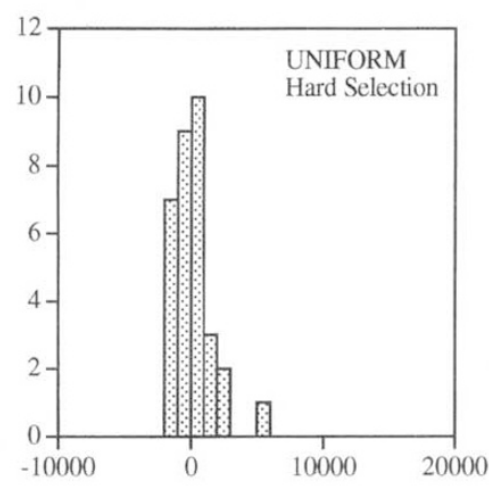

Genetic Variance

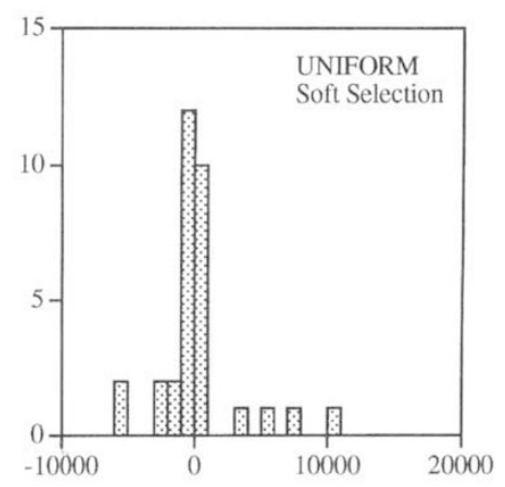

Genetic Variance different concentrations of macronutrients (Bell, 1992), the genetic correlation was found to decline towards zero as the difference between environments increased; genetic correlation was not consistently negative, even for the most extreme contrasts. In this experiment, selection in the short term did not reverse the sign of genetic correlation and, therefore, would not sustain variation at equilibrium. This might not continue to hold in the longer term. Genes with favourable effects in all conditions would tend to be fixed, and those with deleterious effects in all environments lost. Most of the variation remaining would then be contributed by genes with antagonistic effects in different environments, and this variation would be supported by soft selection, although not by hard selection. Thus, it might require a longer-term experiment to distinguish between the effects of soft and hard selection in heterogeneous environments.

Moreover, soft and hard selection might have different consequences if the selection lines were cultured in environments that differed qualitatively, rather than merely in the concentrations of macronutrients. If the genetic correlation among environments were negative in the base population, short-term sorting could maintain variation stably under soft selection.

Perhaps the most striking result of the experiment was the effectiveness of environmental heterogeneity in maintaining high levels of genetic variance in 
Table 3 Genetic variance of uniform selection lines in exotic environments

\begin{tabular}{|c|c|c|c|c|c|c|c|}
\hline \multirow[b]{2}{*}{ Environment } & \multirow[b]{2}{*}{ Selection } & \multirow[b]{2}{*}{$\mathrm{L}$} & \multirow[b]{2}{*}{ S } & \multicolumn{4}{|c|}{ Exotic environment } \\
\hline & & & & TAP & HS & Kuhl & Overall \\
\hline \multirow[t]{4}{*}{ Initial } & \multirow[t]{4}{*}{ None } & 1 & 1 & 2327 & 1261 & $3098^{* *}$ & 2229 \\
\hline & & 1 & 2 & $2323^{*}$ & 4128 & -959 & 1831 \\
\hline & & 2 & 1 & 1275 & $6202^{*}$ & $6031^{*}$ & 4503 \\
\hline & & 2 & 2 & 2242 & $5194^{*}$ & 7704 & 5047 \\
\hline \multirow[t]{4}{*}{ Uniform } & \multirow[t]{4}{*}{ Hard } & 1 & 1 & 1706 & 1060 & -11 & 918 \\
\hline & & 1 & 2 & -164 & 192 & -2581 & -851 \\
\hline & & 2 & 1 & $2489^{*}$ & $5563^{*}$ & 1314 & 3122 \\
\hline & & 2 & 2 & 2207 & $7032^{*}$ & $9217^{* *}$ & 6152 \\
\hline \multirow[t]{4}{*}{ Uniform } & \multirow[t]{4}{*}{ Soft } & 1 & 1 & 43 & -1237 & -2274 & -1156 \\
\hline & & 1 & 2 & 31 & $1904^{*}$ & -304 & 544 \\
\hline & & 2 & 1 & 9292 & 100 & 955 & 3449 \\
\hline & & 2 & 2 & -1835 & -1206 & -1268 & -1436 \\
\hline
\end{tabular}

Data are estimates of genetic variance components from single-classification ANOVA within each environment. The environments are: TAP, Gorman-Levine Tris-acetate-phosphate; HS, Sueoka high-salt medium; Kuhl, Kuhl's medium. They differ from the Bold's medium used as the basis for the the selection environments in various ways: TAP contains ammonium chloride and Tris buffer; HS contains ammonium chloride and much greater quantities of phosphates; Kuhl's has potassium rather than sodium nitrate, but sodium rather than potassium phosphates. Detailed compositions are given by Harris $(1989$, p. 26). Asterisk conventions as in Table 1 . The anomalously high variance expressed by line 2 , sample 1 , of the uniform soft treatment was attributable to the high score of a single spore.

fitness, even under hard selection. The most economical explanation of this result is simply that the directional selection that so rapidly eliminated variance in the uniform environment is obstructed in a heterogeneous environment, so that the loss of genetic variance of fitness is retarded in the short term. It may be that too much emphasis has hitherto been placed on equilibrium theories of diversity. A heterogeneous environment, the composition of which changes from time to time, may support high levels of genetic variance merely because the theoretical end point of selection, the elimination of variation, is never reached. The results of this experiment indicate the importance of time-scale in evaluating the effect of environmental heterogeneity on levels of genetic variance.

\section{Acknowledgements}

This work was supported by a research grant from the Natural Sciences and Engineering Research Council of Canada. I am grateful to Lori Pilkonis for technical assistance.

\section{References}

BELL, G. 1990. The ecology and genetics of fitness in Chlamydomonas. I. Genotype-by-environment interaction among pure strains. Proc. $R$. Soc. Lond. B, 240, 295-321.

BELL, G. 1991. The ecology and genetics of fitness in Chlamydomonas. III. Genotype-by-environment interaction within strains. Evolution, 45, 668-679.

BELL, G. 1992. The ecology and genetics of fitness in Chlamydomonas. $\mathrm{V}$. The relationship between genetic correlation and environmental variance. Evolution, 46, $561-566$.

DEMPSTER, E. R. 1955. Maintenance of genetic heterogeneity. Cold Spring Harb. Symp. Quant. Biol., 20, $25-32$.

HALEY, C. S. AND BIRLEY, A. J. 1983. The genetical response to natural selection by varied environments. II. Observations on replicate populations in spatially varied laboratory environments. Heredity, 51, 581-606.

HARRIS, E. 1989. The Chlamydomonas Source-Book. Academic Press, New York.

HEDRICK, P. W. 1986. Genetic polymorphism in heterogeneous environments: a decade later. Ann. Rev. Ecol. Syst., 17, 535-566.

(c) The Genetical Society of Great Britain, Heredity, 78, 490-497. 
LEVENE, H. 1953. Genetic equilibrium when more than one ecological niche is available. Am. Nat., 87, 331-333.

MAYNARD SMITH, J. AND HOEKSTRA, R. 1980. Polymorphism in a varied environment: how robust are the models? Genet. Res., 35, 260-277.

MINAWA, A. AND BIRLEY, A. J. 1978. The genetical response to natural selection by varied environments. I. Shortterm observations. Heredity, 40, 31-50.

Rosenzweig, M. 1995. Species Diversity in Space and Time. Cambridge University Press, Cambridge.

STROBECK, C. 1974. Sufficient conditions for polymorphism with $\mathrm{N}$ niches and $\mathrm{M}$ mating groups. Am. Nat., 108, 152-156.

Tilman, D. 1982. Resource Competition and Community Structure. Princeton University Press, Princeton, NJ.

VIA, S. AND LANDE, R. 1985. Genotype-environment interaction and the evolution of phenotypic plasticity. Evolution, 39, 505-522.

YAMAZAKI, T., KASEKABE, S., TACHIDA, H., MATSUDA, M. AND MUKAI, T. 1980. Re-examination of diversifying selection of polymorphic allozyme genes by using population cages in Drosophila melanogaster. Proc. Natl. Acad. Sci. U.S.A., 80, 5789-5792. 